\title{
Attention-deficit hyperactivity disorder: treatment discontinuation in adolescents and young adults
}

Suzanne McCarthy, Philip Asherson, David Coghill, Chris Hollis, Macey Murray, Laura Potts, Kapil Sayal, Ruwan de Soysa, Eric Taylor, Tim Williams and Ian C. K. Wong

\section{Background}

Symptoms of attention-deficit hyperactivity disorder (ADHD) are known to persist into adulthood in the majority of cases.

\section{Aims}

To determine the prevalence of methylphenidate, dexamfetamine and atomoxetine prescribing and treatment discontinuation in adolescents and young adults.

\section{Method}

A descriptive cohort study using the UK General Practice Research Database included patients aged 15-21 years from 1999 to 2006 with a prescription for a study drug.

\section{Results}

Prevalence of prescribing averaged across all ages increased 6.23-fold over the study period. Overall, prevalence decreased with age: in 2006, prevalence in males dropped 95\% from 12.77 per 1000 in 15-year-olds to 0.64 per 1000 in 21-year-olds. A longitudinal analysis of a cohort of 44 patients aged 15 years in 1999 demonstrated that no patient received treatment after the age of 21 years.

\section{Conclusions}

The prevalence of prescribing by general practitioners to patients with ADHD drops significantly from age 15 to age 21 years. The fall in prescribing is greater than the reported age-related decrease in symptoms, raising the possibility that treatment is prematurely discontinued in some young adults in whom symptoms persist.

\section{Declaration of interest}

I.C.K.W. was funded by a Department of Health Public Health Career Scientist Award at the time of the study. I.C.K.W., P.A., C.H., K.S. and E.T. are members of the National Institute for Health and Clinical Excellence guideline committee on ADHD. P.A. has attended advisory board meetings for Janssen-Cilag and Shire and has been reimbursed for talks at Janssen-Cilag, Eli Lilly and UCB Pharma sponsored meetings. D.C. is an advisory board member for Cephalon, Eli Lilly, Janssen Cilag, Shire and UCB-Celltech, and has research funding from Eli Lilly and Janssen-Cilag; he is on the professional board of the National Attention Deficit Disorder Information and Support Service (ADDISS) and is on the project group for the NHS Quality Improvement Scotland audit of ADHD care in Scotland. K.S. has received reimbursement of expenses by Janssen-Cilag, manufacturer of methylphenidate, for attending a conference. R.D.S. has been reimbursed by Janssen-Cilag, UCB Pharma and Lilly Pharmaceuticals, manufacturer of methylphenidate and atomoxetine, for attending several conferences, and has been paid by UCB Pharma for attending consultation workshops. The School of Pharmacy, University of London has received an educational grant from Janssen-Cilag.
Attention-deficit hyperactivity disorder (ADHD) is a common neurodevelopmental disorder affecting $3-5 \%$ of children in the $\mathrm{UK}^{1,2}$ It was once considered to be a condition confined to childhood, and indeed previous National Institute for Health and Clinical Excellence (NICE) guidelines from 2000 recommended that treatment with methylphenidate should normally be stopped in adolescence. ${ }^{2}$ There is, however, increasing evidence that the core symptoms persist into adulthood and are associated with continued clinical and psychosocial impairments. ${ }^{3,4}$ There are now guidelines in the UK on how ADHD should be treated in older adolescents and young adults, ${ }^{5,6}$ including a revised recommendation from NICE for use of stimulant medication in adults with a diagnosis of ADHD. ${ }^{7}$ Premature cessation during adolescence may impair function at a crucial developmental stage. Untreated ADHD is associated with several negative outcomes in adulthood including poor rates of employment, harm to relationships with family and friends, increased rates of criminality and accidents, and the development of comorbid psychiatric symptoms including anxiety, depression and substance misuse. ${ }^{8,9}$ In recent years, the National Health Service (NHS) Health Technology Assessment Research Programme has called for more research to guide the appropriate cessation of ADHD treatments in older adolescents and young adults; however, it is not clear to what extent this patient group currently persists with drug therapy. The aims of our study were to determine the prevalence of methylphenidate, dexamfetamine and atomoxetine prescribing and to investigate discontinuation patterns in adolescents and young adults.

\section{Method}

\section{Data source}

The data for this study were obtained from the General Practice Research Database (GPRD). This is a computerised database of anonymised longitudinal patient records which is maintained by the Medicines and Healthcare Products Regulatory Agency. The database has previously been used to investigate paediatric psychotropic medication prescribing in the $\mathrm{UK},{ }^{10-12}$ including an investigation of the prevalence and incidence of drug treatment of ADHD in younger boys in $1999 .{ }^{13}$ At present the GPRD contains data for 3 million active patients (about $5 \%$ of the UK population) with a demographic distribution similar to the UK population. Participating general practitioners enter data on patients including demographic details, diagnoses, prescriptions and hospital referrals. Validation studies show that the quality and completeness of the data are high. ${ }^{14-16}$ Approval for the study was granted by the GPRD's independent scientific and ethical advisory committee.

Our study period was 1 January 1999 to 31 December 2006. For eligibility to enter the study, patients had to be aged between 15 
years and 21 years during the study period with at least one prescription for methylphenidate (immediate and modified-release preparations), dexamfetamine or atomoxetine. These three drugs were chosen as they are the only medications currently licensed in the UK for the treatment of ADHD and are used almost exclusively for this purpose.

\section{Discontinuation pattern (drug cessation)}

A cross-sectional analysis was carried out by identifying all prescriptions for a study drug issued during the study period for patients aged 15-21 years. Age- and gender-specific prevalence rates were calculated. The sample size for this analysis was 1636 patients. Prevalence was defined as the number of patients with one or more prescriptions for the study drugs per 1000 patients in the population. Trends in annual prevalence from 1999 to 2006 were examined using the chi-squared test for trend. Data were analysed using Stata/SE version 9.1 for Windows.

A longitudinal analysis was then conducted in a cohort of patients to determine duration and cessation of treatment. All patients in the drug cohort who were aged 15 years in 1999 ( $n=44)$ were followed from 1 January 1999 to 31 December 2006. This cohort was chosen to enable a follow-up within the study period of patients aged 15 years until they reached the age of 21 years. Patients who stopped treatment were identified by screening for any records of treatment cessation. A minimum duration of 6 months from the last prescription issued was indicative of treatment cessation. The duration of a prescription was calculated from dividing the quantity of medication prescribed by the daily dosage, and in turn, overall treatment duration was determined from the date of the first prescription to the end date of the last prescription ever recorded on the database. For some patients who might have stopped and restarted treatment during the inclusion time frame, only the total duration of treatment from the first to the last recorded prescription was considered. Duration, therefore, included intervals of no treatment, which could lead to an overestimate of the total duration of treatment. Kaplan-Meier analysis was used to estimate cessation of treatment in the target group.

We predicted that the rate of decline in prescriptions for ADHD would mirror the expected rate of decline in diagnostic prevalence. The GPRD does not contain data from which diagnostic prevalence at each age can be calculated, and so we estimated the decline in diagnostic prevalence using published data from the meta-analysis of follow-up studies conducted by Faraone et al. ${ }^{4}$ They examined the persistence of ADHD into adulthood using only data from high-quality, well-designed published follow-up studies which allowed a distinction to be made between people with syndromatic and symptomatic persistence and also between these individuals and those in full remission. From these data the probability of persistence of symptoms associated with a 1-year increase in age was calculated to be $83 \%$ for patients meeting full criteria (syndromatic persistence) and $96 \%$ for those with residual symptoms (symptomatic persistence) of ADHD. Using the more conservative figure of $83 \%$ for each 1 -year change in age (i.e. patients who retain the full DSM diagnosis); we should expect to see an equivalent reduction in prescribing rates of around $17 \%$ each year.

\section{Results}

During the study period, 22013 prescriptions were issued to 1636 patients, of whom 1452 (89\%) were male. The overall prevalence of prescriptions in this sample of patients increased 6.23-fold between 1999 and 2006. In 1999, the prevalence of drug prescribing for male patients aged 15-21 years was 0.88 per
1000 patients (95\% CI $0.72-1.08$ ), whereas in 2006 the prevalence per 1000 patients was 5.09 (95\% CI 4.73-5.47). This was an overall 5.78 -fold increase in prevalence over the 8 -year period $\left(\chi^{2}=705.7\right.$, d.f. $=1, P<0.001$ for trend). In female patients in 1999 the prevalence was 0.06 per 1000 patients (95\% CI 0.02-0.12); however, in 2006 this figure rose to 0.77 (95\% CI $0.63-0.93$ ), a 12.83 -fold increase ( $\chi^{2}=147.4$, d.f. $=1, P<0.001$ for trend). These data show that the gender difference in treatment prevalence declined from approximately $14.7: 1$ to $6.6: 1$ (male:female) over this period.

Figure 1 illustrates the change in prescription rates for males aged 15-21 years between 1999 and 2006. Fitting a logistic regression model with grouped data including age, year and an age $\times$ year interaction as continuous variables, it was demonstrated that increasing age significantly decreased treatment prevalence $(P<0.001)$. The figure indicates an age $\times$ year interaction (odds ratio $1.02,95 \%$ CI 1.01-1.03, $P=0.001$ ) with a marked increase in prevalence for younger adolescents, but almost no increase in the prescription prevalence for older adolescents and young adults. In the most recent year surveyed in this study (2006) the data show that the prescription prevalence for 21year-old males was $95 \%$ lower than that for 15-year-old males (12.77 per 1000 patients compared with 0.64 per 1000 patients). The $\chi^{2}$-test for trend showed a significant linear trend $(P<0.001)$, demonstrating a strong effect of age on decreasing treatment prevalence.

In order to address the issue of discontinuation more directly, a longitudinal study was also conducted on the cohort of patients who were 15 years old in 1999. Forty-four patients (43 male) were identified and the prescriptions of these patients were mapped to determine duration of treatment. Kaplan-Meier analysis was used to estimate the proportion of patients in the cohort group continuously treated from age 15 to age 21 years (Fig. 2). The median duration of medication treatment for the cohort was 1.80 years (95\% CI 1.04-2.56) and no patient remained on treatment beyond the age of 21 years. The rate of persistence with treatment falls well below the expected rate.

\section{Discussion}

To our knowledge this is the first study to examine prescribing trends of methylphenidate, dexamfetamine and atomoxetine in adolescents and young adults in primary care in the UK. There are four key findings. First, there was a marked rise over time, combining age groups, in the prescribing of stimulants and atomoxetine in adolescents and young adults, with an overall 6.23 -fold increase in prevalence over the 8-year period between 1999 and 2006. Second, although over this same period the rate of prescribing in females has increased at a greater rate than that

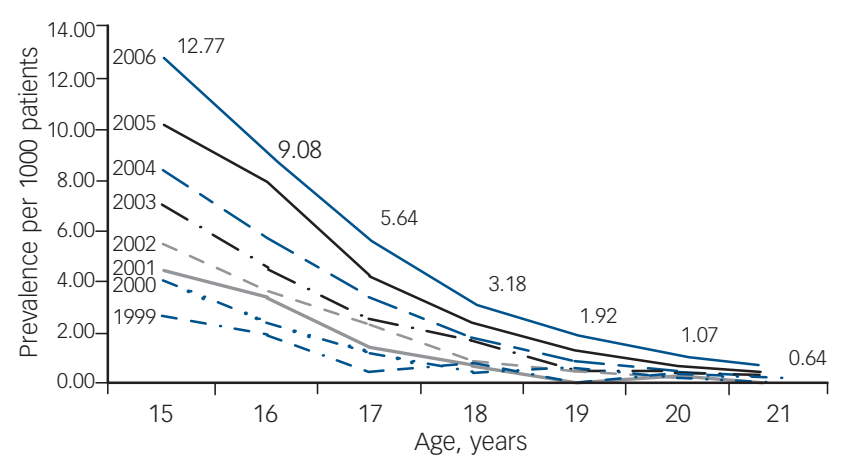

Fig. 1 Prevalence of prescribing of methylphenidate, dexamfetamine and atomoxetine to male patients from age 15 years to age 21 years, 1999-2006. 


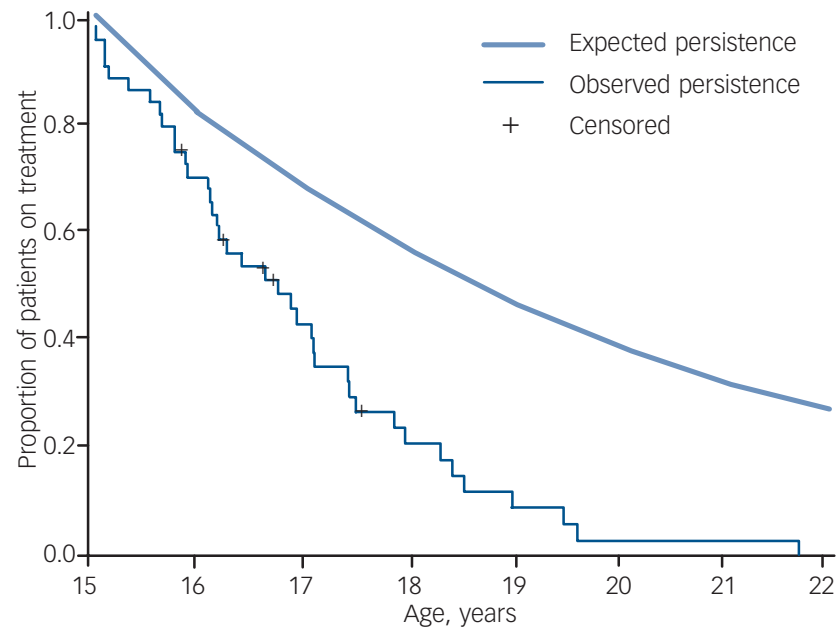

Fig. 2 Proportion of patients aged 15 years in 1999 remaining in treatment for each 1-year change in age $(n=44)$ (expected persistence $83 \%$ ).

in males, the ratio of males to females receiving prescriptions (6.6:1) remains higher than the $4: 1$ gender ratio for ADHD in population samples. ${ }^{17}$ This indicates that females with ADHD are still less likely to be identified and/or treated with medication than males. Third, the cross-sectional analysis showed an interaction with age with a greater increase in prescribing in younger patients. Fourth, the longitudinal cohort analysis demonstrated discontinuation of prescribing in older adolescents and young adults, with no patient still receiving treatment by age 21 years. There are several possible explanations for these findings.

\section{Early discontinuation of medication}

The overall trend of increased prescribing over the study period may be attributed to increased recognition and treatment of ADHD by child and adolescent mental health and paediatric services, in addition to the increased marketing and availability of drugs to treat ADHD (e.g. long-acting methylphenidate and the non-stimulant atomoxetine). In contrast, the data indicate that there was no parallel increase in the rates of prescribing to older adolescents and young adults. Furthermore, since prescription rates show such a rapid tail-off in young adults, it is likely that in most cases prescriptions for individual patients with ADHD are tailed off and stopped during late adolescence and early adult years. The best evidence for this is the Kaplan-Meier analysis data (Fig. 2), which shows that all patients in the cohort followed from age 15 years in 1999 stopped treatment by age 21 years.

An important question is whether the low level of prescribing for young adults is appropriate and matches the clinical course of the disorder. The pattern of treatment discontinuation seen in the cohort study would be appropriate were ADHD a time-limited condition confined to childhood and adolescence, or alternatively were drug treatment not effective in adults. The main evidence against this view comes from longitudinal follow-up studies of ADHD that show high levels of persistence of the core ADHD syndrome and associated impairments. A recent meta-analysis found that $15 \%$ of children with ADHD continued to fulfil the full criteria for ADHD as adults by age 25 years. ${ }^{4}$ This is significant because the individuals with persistent ADHD fulfilled the same diagnostic criteria that are applied to children, which represents a significant level of impairment compared with age- and gender-matched controls. Furthermore, the meta-analysis data found a high level of impairment in individuals who no longer met full criteria for ADHD but were in partial remission, with a lower symptom count. Unfortunately, this meta-analysis was unable to take medication status into account as these data were not available for all of the included studies. That impairments exist in the group of people with ADHD that persists into adulthood is well documented in the follow-up studies as well as from reports of epidemiological surveys. ${ }^{9,18}$ Findings from meta-analyses also suggest that the stimulant drug methylphenidate is equally effective in reducing ADHD symptoms in adults as it is in children. ${ }^{19,20}$ Both the stimulant and nonstimulant medications have been demonstrated to be efficacious and effective at reducing the symptoms and impairments associated with ADHD in adults, with effect sizes of around 0.9 for the stimulants and 0.6 for the non-stimulant atomoxetine. ${ }^{19,21}$

Based on a thorough review of the literature and expert opinion on the treatment of ADHD in adults, the British Association of Psychopharmacology concluded that:

'it is becoming increasingly evident that this common and impairing condition is costly and treatable, providing a significant opportunity to relieve the burden of suffering from patient and family, but also to alleviate social costs in unemployment, crime, incarceration, smoking, substance use and driving accidents. ${ }^{\prime}$

Furthermore, one of their main recommendations is that it is appropriate to treat $\mathrm{ADHD}$ in adults in the same way that the disorder is treated in children. This is the same conclusion reached by the most recent NICE guideline development group. ${ }^{7}$

Several factors appear to contribute to the low level of prescribing with increasing age. First, the steepest decrease in prescribing occurred between the ages of 16 and 17 years. At this age, adolescents normally finish their General Certificate of Secondary Education and may leave school. This might be critical since the school system is known to have a key role in the identification and referral of young people with ADHD, ${ }^{17}$ and after leaving school young people may perceive less need for sustained attention and focus or control over hyperactive-impulsive behaviour. Furthermore, there may be less expectation from key adults (teachers and parents) that treatment is still necessary. Some patients develop coping strategies as they grow older to compensate for the impairments caused by ADHD. In a separate but related Health Technology Assessment-funded study conducted by the authors, interviews with patients with ADHD and clinicians involved in the care of patients with ADHD revealed that some patients who stopped treatment did so because they felt they were able to control themselves better than they could when they were younger. Others had gravitated towards environments and employment that did not place the same demands on maintaining focus and concentration. ${ }^{22}$ Another factor is that young people themselves have greater autonomy in making decisions about their healthcare, and problems with self-evaluation and adherence to treatment regimens are recognised problems in this age group across many medical conditions. For example, the increase in self-autonomy during adolescence is often accompanied by poor drug adherence, typically seen in conditions such as diabetes. ${ }^{23}$ Second, the low level of prescribing is accompanied by the poor provision of diagnostic and treatment services for older adolescents and young adults. Typically in the UK both paediatric and child and adolescent mental health services are available for young people up to the age of 16 years or school-leaving age. ${ }^{24}$ However, ADHD services within adult mental health are currently poorly developed $^{25}$ and clear arrangements for transition are often lacking. ${ }^{26}$ This can result in patients failing to be picked up by adult services for initiation or continuation of treatment for ADHD, even where this is clinically indicated. ${ }^{22}$ The further recommendation that prescriptions of stimulants and atomoxetine should only be provided under the supervision of a clinician with expertise in ADHD is problematic within adult mental health services where specialist services are limited. 
Currently in the UK neither methylphenidate nor dexamfetamine are licensed for the treatment of ADHD in patients over 18 years old and atomoxetine is only licensed for individuals over the age of 18 years who started their treatment before that age. As previously noted, the recommendation by NICE in their 2000 guideline was that treatment should be stopped during adolescence. $^{2}$ This advice has been removed from the recently published NICE guidelines, ${ }^{7}$ which in contrast highlight the need for continued treatment in a proportion of cases.

One may argue that in the UK the relatively low level of prescribing to older patients is due to inappropriate overprescribing in the younger age group; therefore, clinicians decide to stop treatment when patients are older. However, based on our findings and existing data, ${ }^{13}$ this argument cannot be substantiated. In our cohort in 1999, the prevalence of prescribing in males aged 15 years was less than 3 per 1000 patients, which is far lower than the expected prevalence of children with ADHD or hyperkinetic disorders in the UK, estimated to be $5 \%$ and $1 \%$ in respectively. ${ }^{2}$ A recent national survey also concluded that concerns about overprescribing of stimulant medications in the UK were unfounded. ${ }^{27}$ This survey found that all children aged 5-16 years receiving stimulant treatment had evidence of pervasive hyperactivity (overactivity, impulsiveness and inattention). Despite this, a large proportion of children (about $57 \%$ ) with hyperkinetic disorders, which represent a severe form of the DSM-IV ADHD diagnosis, were not getting access to an evidence-based treatment. Similar findings were reported by the NHS Quality Improvement Scotland review of ADHD treatment by NHS services across Scotland, which found that only $0.7 \%$ of the children in Scotland were currently being treated for ADHD. ${ }^{26}$ This problem appears to be further exacerbated in older adolescents and young adults.

\section{Strengths and weaknesses of the study}

The GPRD is one of the largest databases of anonymised longitudinal data from primary care in the world, capturing comprehensive information on treatments and outcomes from a $5 \%$ sample of British general practices. The use of the GPRD allowed us to capture what is actually happening under normal conditions of practice, rather than in selected samples of patients recruited into clinical trials. There are, however, a number of limitations in using this database. It does not record information concerning treatment indications, dispensing of prescriptions or treatment adherence (a limitation of many automated databases). Although our study is a true reflection of primary care, it may underestimate the true prevalence of ADHD treatment in the $\mathrm{UK}$, as some general practitioners are unwilling to prescribe treatments for ADHD for various reasons. Prescribing is then done solely in secondary or tertiary care, but there is no information to show the proportion of patients in whom this occurs. Nevertheless, many patients will be prescribed treatment from their general practitioner under a shared care protocol, following diagnosis and initiation of treatment from a child and adolescent psychiatrist or paediatrician. Although this study shows discontinuation of prescribing to patients by general practitioners, we do not assume that these doctors alone are taking the decision to stop medication, because under the NICE 2000 guidance treatment discontinuation should occur under specialist supervision. It is possible that young people no longer request prescriptions or attend follow-up for monitoring. It is also possible that patients were receiving other forms of therapy for ADHD that were not captured in this study. These might have included other pharmacological treatments not licensed for treating ADHD such as clonidine, guanfacine, modafinil and bupropion, or the antipsychotics and antidepressants used by some practitioners despite a lack of evidence for their efficacy. The NICE guidelines on ADHD (in the public consultation phase at the time of writing) state that where drug treatment is considered appropriate, methylphenidate, dexamfetamine and atomoxetine are recommended as first-line treatments, within their licensed indications. ${ }^{7}$ It would not have been possible with our study method to have investigated the use of these other medications in the treatment of ADHD, because unlike methylphenidate, dexamfetamine and atomoxetine they are frequently prescribed for conditions other than ADHD.

\section{Unanswered questions and future research}

It has to be acknowledged that there is a lack of good-quality trial data in patients of all ages to provide direct evidence that the benefits of medication treatment continue when this treatment is used over the medium to long term. Unfortunately, even studies with long-term follow-up of treatment response such as the Multimodal Treatment of ADHD study were not designed to answer this question. ${ }^{28,29}$ Therefore, future research should focus on determining the long-term efficacy, effectiveness and safety of ADHD medications in both children and adults. Following this, further research would be required to examine the process of treatment continuation and discontinuation so that patients and clinicians can make an informed decision whether to continue or stop treatments beyond childhood.

Qualitative studies with adolescents to investigate attitudes to ADHD medication and health services research into transition services for ADHD are needed to determine whether evidencebased interventions are accessible. Finally, further studies should be conducted to examine the effectiveness of behavioural and psychosocial interventions in treating patients whose symptoms persist but who wish to discontinue long-term drug treatment.

\section{Implications of the study}

Since 1999, the prevalence of drug prescribing for adolescents and young adults with ADHD has increased rapidly; but the rise in prevalence is lower as the patients become older. There is a marked pattern of drug discontinuation between the ages of 15 and 21 years, with almost all patients having discontinued treatment in early adult life. This study raises the possibility that treatment may be prematurely discontinued by or for some adolescents and young adults with ADHD and that overall the relative decline in treatment prevalence may be out of step with the number of people who still require treatment as young adults. Therefore, further research should target reasons behind medication cessation and the appropriate management of these patients.

Suzanne McCarthy, Centre for Paediatric Pharmacy Research, School of Pharmacy, University of London and Institute of Child Health, University College London; Philip Asherson, MRC Social, Genetic and Developmental Psychiatry Centre Institute of Psychiatry, London; David Coghill, Section of Psychiatry, University of Dundee, Ninewells Hospital and Medical School, Dundee; Chris Hollis, Division of Psychiatry, School of Community Health Sciences, Nottingham; Macey Murray, Centre for Paediatric Pharmacy Research, School of Pharmacy, University of London, and Institute of Child Health, University College London; Laura Potts, Mental Health and Neuroscience Clinical Trial Unit, Institute of Psychiatry, London; Kapil Sayal, Division of Psychiatry, School of Community Health Sciences, Nottingham; Ruwan de Soysa, Royal Liverpool Children's NHS Trust, Alder Hey, Liverpool; Eric Taylor, Department of Child and Adolescent Psychiatry, Anstitute of Psychiatry, London; Tim Williams, General Practice Research Database, Medicines and Healthcare Products Regulatory Agency, London; Ian C. K. Wong, Centre for Paediatric Pharmacy Research, School of Pharmacy, University of London and Institute of Child Health, University College London, UK

Correspondence: Ian C. K. Wong, Institute of Child Health, University College London, 29/39 Brunswick Square, London WC1N 1AX, UK. Email: ian.wong@pharmacy.ac.uk

First received 8 Nov 2007, final revision 28 Jul 2008, accepted 1 Sep 2008 


\section{Acknowledgements}

This project was funded by the National Health Service Research and Development Health Technology Assessment Programme (project number 04/36/02). The licence for the General Practice Research Database was funded by the European Commission via the Taskforce European Drug Development for the Young (TEDDY) Network of Excellence unde the European Commission Framework 6 Programme 2005-2010. The views and opinions expressed in this paper are those of the authors and do not necessarily reflect those of the Department of Health.

\section{References}

1 Ford T, Goodman R, Meltzer, H. The British Child and Adolescent Mental Health Survey 1999: the prevalence of DSM-IV disorders. J Am Acad Child Adolesc Psychiatry 2003; 42: 1203-11.

2 National Institute for Health and Clinical Excellence. Guidance on the Use of Methylphenidate (Ritalin, Equasym) for Attention Deficit/Hyperactivity Disorder (ADHD) in Childhood. Technology Appraisal Guidance 13. NICE, 2000.

3 Faraone SV, Biederman J, Spencer T, Wilens T, Seidman LJ, Mick E, et al. Attention-deficit/hyperactivity disorder in adults: an overview. Biol Psychiatry 2000; 48: 9-20.

4 Faraone SV, Biederman J, Mick E. The age-dependent decline of attention deficit hyperactivity disorder: a meta-analysis of follow-up studies. Psycho Med 2006; 36: 159-65.

5 Nutt DJ, Fone K, Asherson P, Bramble D, Hill P, Matthews K, et al. Evidencebased guidelines for management of attention-deficit/hyperactivity disorder in adolescents in transition to adult services and in adults: recommendations from the British Association for Psychopharmacology. J Psychopharmacol 2006; 21: 10-41.

6 Asherson P. Clinical assessment and treatment of attention deficit hyperactivity disorder in adults. Expert Rev Neurother 2005; 5: 525-39.

7 National Institute for Health and Clinical Excellence. Attention Deficit Hyperactivity Disorder: Diagnosis and Management of ADHD in Children, Young People and Adults. NICE, 2008.

8 Nahlik J. Issues in diagnosis of attention-deficit/hyperactivity disorder in adolescents. Clin Pediatr 2004; 43: 1-10.

9 Kessler RC, Adler L, Barkley R, Biederman J, Conners CK, Demler O, et al. The prevalence and correlates of adult ADHD in the United States: results from the National Comorbidity Survey Replication. Am J Psychiatry 2006; 163 : 716-23.

10 Murray ML, de Vries CS, Wong ICK. A drug utilisation study of antidepressants in children and adolescents using the General Practice Research Database. Arch Dis Child 2004; 89: 1098-102.

11 Ackers R, Murray ML, Besag FM, Wong IC. Prioritising children's medicines for research: a pharmacoepidemiological study on antiepileptic drugs. $\mathrm{Br} J \mathrm{Clin}$ Pharmacol 2007; 63: 689-97.

12 Rani F, Murray M, Byrne P, Wong IC. Epidemiology of antipsychotic prescribing to children and adolescents in UK primary care. Pediatrics 2008; 121: 1002-9.
13 Jick H, Kaye JA, Black C. Incidence and prevalence of drug-treated attention deficit disorder among boys in the UK. Br J Gen Pract 2004; 54: 345-47.

14 Hollowell J. The General Practice Research Database: quality of morbidity data. Popul Trends 1997; 87: 36-40.

15 Wong IC, Murray ML. The potential of UK clinical databases in enhancing paediatric medication research. Br J Clin Pharmacol 2005; 59: 750-5.

16 Walley T, Mantgani A. The UK General Practice Research Database. Lancet 1997; 350: 1097-9.

17 Sayal K, Goodman R, Ford T. Barriers to the identification of children with attention deficit/hyperactivity disorder. J Child Psychol Psychiatry 2006; 47 $744-50$

18 Fayyad J, De Graaf R, Kessler R, Alonso J, Angermeyer M, Demyttenaere K, et al. Cross-national prevalence and correlates of adult attention-deficit hyperactivity disorder. Br J Psychiatry 2007; 190: 402-9.

19 Faraone SV, Spencer T, Aleardi M, Pagano C, Biederman J. Meta-analysis of the efficacy of methylphenidate for treating adult attention-deficit/ hyperactivity disorder. J Clin Psychopharmacol 2004; 24: 24-9.

20 Faraone SV, Biederman J, Spencer TJ, Aleardi M. Comparing the efficacy of medications for ADHD using meta-analysis. MedGenMed 2006; 8: 4.

21 Michelson D, Adler L, Spencer T, Reimherr FW, West SA, Allen AJ, et al Atomoxetine in adults with ADHD: two randomized, placebo-controlled studies. Biol Psychiatry 2003; 53: 112-20.

22 Wong ICK, Asherson P, Clifford S, Coghill D, DeSoysa R, Hollis C, et al. Cessation of attention deficit hyperactivity disorder drugs in the young (CADDY). Health Technol Assess (in press).

23 Miller VA, Drotar D. Decision-making competence and adherence to treatment in adolescents with diabetes. J Pediatr Psychol 2007; 32: 178-88.

24 National Service Framework for Children, Young People and Maternity Services. Transition: Getting it Right for Young People. Improving the Transition of Young People with Long-Term Conditions from Children's to Adult Health Services. UK Department of Health, 2006 (http:// www.dh.gov.uk/assetRoot/04/13/21/49/04132149.pdf)

25 Asherson $\mathrm{P}$, Chen W, Craddock B, Taylor E. Adult attention-deficit hyperactivity disorder: recognition and treatment in general adult psychiatry. Br J Psychiatry 2007; 190: 4-5.

26 NHS Quality Improvement Scotland. ADHD - Services Over Scotland. Report of the Service Profiling Exercise. NHS OIS, 2007 (http://www. nhshealthquality.org/nhsqis/files/ADHD_ServicesOverScotland_MAR07.pdf).

27 Green H, McGinnity A, Meltzer H, Ford T, Goodman R. Hyperkinetic disorders. In Mental Health of Children and Young People, Great Britain 2004. Palgrave Macmillan, 2005.

28 Jensen PS, Arnold LE, Swanson JM, Vitiello B, Abikoff HB, Greenhill LL, et al. 3-year follow-up of the NIMH MTA study. J Am Acad Child Adolesc Psychiatry 2007; 46: 989-1002.

29 Swanson JM, Hinshaw SP, Arnold LE, Gibbons RD, Marcus S, Hur K, et al. Secondary evaluations of MTA 36-month outcomes: propensity score and growth mixture model analyses. J Am Acad Child Adolesc Psychiatry 2007 46: 1003-14. 